\title{
On the optimal control problem governed by quasistationary von Kárman's equations
}

\author{
Igor Bock \\ Department of Mathematics, FEI STU \\ Ilkovičova 3, 812 19 Bratislava, Slovakia. \\ $E$-mail: bock@kmat.elf.stuba.sk \\ and \\ Ján Lovíšek \\ Department of Mechanics, Faculty of Constructions STU \\ Radlinského 11, 81368 Bratislava, Slovakia
}

\begin{abstract}
Optimal control problems with evolutionary generalized Von Kárman's equations in the role of a state problem are considered. The existence theorems for the state and the control problem are stated. The necessary optimality conditions are investigated.
\end{abstract}

Keywords

Quasistationary von Kárman's equations, Volterra integral equation, optimal control problem, necessary optimality conditions

\section{INTRODUCTION}

Optimal control problems for stationary Von Kárman's equations were investigated in the joint papers of (Bock, Hlaváček and Lovíšek, 1984, 1985, 1987) Here we consider in the role of state equations evolutionary Von Kárman's equations describing large deflections of thin viscoelastic plates. The first part of the paper is devoted to the deriving and solving the state problem, whose canonical form is a nonlinear pseudoparabolic equation with an integro-differential part. Optimal control problems with controls in the right-hand sides are solved in the second part.

\section{FORMULATION OF THE STATE PROBLEM}

Let us assume a thin viscoelastic plate made of a short memory material of the Voigt type (Brilla, 1973), (Christensen, 1971). It occupies the domain

$Q=\left\{(x, z) \in R^{3} ; x=\left(x_{1}, x_{2}\right) \in \Omega,-h / 2<z<h / 2\right\}$, 
where $\Omega$ is a bounded simply connected domain in $R^{2}$ with a Lipschitz boundary $\partial \Omega$. After the linearization of the Kirchhoff hypothesis we obtain the strain-displacement relations

$$
\begin{aligned}
& \varepsilon_{i j}=\frac{1}{2}\left(\partial_{i} \omega_{j}+\partial_{j} \omega_{i}+\partial_{i} y \partial_{j} y\right)-z \partial_{i j} y ; \quad i, j=1,2 \\
& \varepsilon_{13}=\varepsilon_{23}=0, \quad \varepsilon_{33}=\frac{1}{2}\left[\left(\partial_{1} y\right)^{2}+\left(\partial_{2} y\right)^{2}\right]
\end{aligned}
$$

where $\left(\omega_{1}, \omega_{2}\right)$ is the plane displacement vector, $y$ is the deflection of the middle surface of the plate and

$$
\partial_{i} \omega_{j}=\frac{\partial \omega_{j}}{\partial x_{i}}, \quad \partial_{i j} y=\frac{\partial y}{\partial x_{i} \partial x_{j}}
$$

The viscoelastic stress-strain relations have the form

$$
\begin{aligned}
& \sigma^{i j}(t)=A_{i j k l}^{(1)} \partial_{t} \varepsilon_{k l}(t)+A_{i j k l}^{(0)} \varepsilon_{k l}(t), \quad i, j, k, l \in\{1,2\} ; \\
& \sigma^{33}=0
\end{aligned}
$$

The third order tensors $A_{i j k l}^{(r)}$ are symmetric and positively definite

$$
\begin{aligned}
& A_{i j k l}^{(r)}=A_{j i k l}^{(r)}=A_{k l i j}^{(r)} \\
& A_{k l i j}^{(r)} \tau_{i j} \tau_{k l} \geq c_{r} \tau_{i j} \tau_{i j}, \quad c_{r}>0
\end{aligned}
$$

for all $\left\{\tau_{i j}\right\} \in R_{s y m}^{4}$ and $r=0,1$.

Let us introduce the matrices

$$
\mathbf{A}_{r}=\left(\begin{array}{lll}
A_{1111}^{(r)}, & A_{1112}^{(r)}, & A_{1122}^{(r)} \\
A_{1211}^{(r)}, & A_{1212}^{(r)}, & A_{122}^{(r)} \\
A_{2211}^{(r)}, & A_{2212}^{(r)}, & A_{2222}^{(r)}
\end{array}\right), \quad r=0,1 ;
$$

and

$$
\mathbf{G}(t)=\exp \left(-\mathbf{A}_{1}^{-1} \mathbf{A}_{0} t\right) \mathbf{A}_{1}^{-1}
$$

We recall that $\exp \mathbf{A} t$ is the matrix exponential function. The matrix $\mathbf{G}(t)$ is regular and symmetric due to the expansion

$$
\mathbf{G}(t)^{-1}=\mathbf{A}_{1} \exp \left(\mathbf{A}_{1}^{-1} \mathbf{A}_{0} t\right)=\mathbf{A}_{0}+\sum_{k=1}^{\infty} \frac{t^{k}}{k !} \mathbf{A}_{1}\left(\mathbf{A}_{1}^{-1} \mathbf{A}_{0}\right)^{k}
$$


We express it in the form

$\mathbf{G}(t)=\left(\begin{array}{lll}G_{1111}, & G_{1112}, & G_{1122} \\ G_{1211}, & G_{1212}, & G_{1222} \\ G_{2211}, & G_{2212}, & G_{2222}\end{array}\right)$

Assuming that the plate is clamped and the forces acting on the parts $\Omega \times\{-h / 2\}$ and $\Omega \times\{h / 2\}$ are vectors $(0,0,0)$ and $(0,0, v(t, x))$ respectively, we obtain employing the principle of virtual displacements the integro-differential equation connecting the Airy stress function $\Phi$ and the perpendicular deflection $y$ :

$\int_{0}^{t} H_{i j k l}(t-s) \partial_{i j k l} \Phi(s) d s=-\frac{1}{2} h[y, y]$

where

$$
\begin{aligned}
& H_{i j k l}(t)=H_{j i k l}(t)=H_{l k i j}(t), \\
& H_{1111}(t)=G_{2222}(t), \quad H_{1112}(t)=-\frac{1}{2} G_{2212}(t) \\
& H_{1222}(t)=-\frac{1}{2} G_{1112}(t), \quad H_{1122}(t)=\frac{1}{2} G_{1122}(t) \\
& H_{1212}(t)=\frac{1}{4} G_{1212}(t), \quad H_{2222}(t)=G_{1111}(t)
\end{aligned}
$$

and

$$
[w, y]=\partial_{11} w \partial_{22} y+\partial_{22} w \partial_{11} y-2 \partial_{12} w \partial_{12} y
$$

(13) is the Volterra integro-differential equation of the first kind with respect to $\Phi$. After differentiating it with respect to $t$ we obtain the boundary value problem for the Volterra equation of the second kind

$$
\begin{aligned}
& H_{i j k l}(0) \partial_{i j k l} \Phi(t)+\int_{0}^{t} \partial_{t} H_{i j k l}(t-s) \partial_{i j k l} \Phi(s) d s=-h\left[\partial_{t} y, y\right] \\
& \Phi(t, \xi)=\frac{\partial \Phi}{\partial \mathbf{n}}(t, \xi)=0, \quad t>0, \quad \xi \in \partial \Omega
\end{aligned}
$$

In a similar way as in the elastic case the initial-boundary value problem for the pseudoparabolic equation with respect to the deflection $y \equiv y(t, x)$ can be derived :

$$
\begin{aligned}
& \frac{h^{3}}{12}\left(A_{i j k l}^{(1)} \partial_{t} \partial_{i j k l} y+A_{i j k l}^{(0)} \partial_{i j k l} y\right)-[\Phi, y]=v, \quad t>0, \quad x \in \Omega \\
& y(0, x)=y(t, \xi)=\frac{\partial y}{\partial \mathbf{n}}(t, \xi)=0, \quad t>0, \quad \xi \in \partial \Omega
\end{aligned}
$$


The initial-boundary value problem for the system (17) - (20) represents the strong formulation of the generalized Von Kárman's equations for a viscoelastic plate.

In order to solve it we proceed with a weak formulation of the problem in a Sobolev space $V=H_{0}^{2}(\Omega)$. Let us introduce the operators $A_{r}, H(t): V \rightarrow V^{*}\left(V^{*}\right.$ is a dual space of $V$ ) by

$$
\begin{aligned}
& \left\langle A_{r} y, w\right\rangle=\frac{h^{3}}{12} \iint_{\Omega} A_{i j k l}^{(r)} \partial_{i j} y \partial_{k l} w d x ; \quad y, w \in V ; \quad r=0,1 \\
& \langle H(t) y, w\rangle=\iint_{\Omega} H_{i j k l}(t) \partial_{i j} y \partial_{k l} w d x ; \quad y, w \in V
\end{aligned}
$$

The problem (17) - (20) has then a following weak formulation:

For arbitrary $T>0$ find a couple $\{\Phi, y\}:[0, T] \rightarrow V \times V$ fulfilling

$$
\begin{aligned}
& H(0) \Phi(t)+\int_{0}^{t} \partial_{t} H(t-s) \Phi(s) d s=-h\left[\partial_{t} y(t), y(t)\right] \\
& A_{1} \partial_{t} y(t)+A_{0} y(t)-[\Phi(t), y(t)]=v(t) \\
& y(0)=0
\end{aligned}
$$

The operator $H(t)$ is for every $t \in[0, T]$ symmetric and positively definite. Moreover it fulfils the assumptions of Corollary 4.1. from (MacCamy and Wong,1972) and it is nonnegative in the following convolutive sense

$\int_{0}^{T}\left\langle\int_{0}^{t} H(t-s) \Phi(s) d s, \Phi(t)\right\rangle d t \geq 0$

for every $T>0$ and $\Phi(.) \in C([0, T], V)$,

where $C([0, T], V)$ is the set of all continuous functions defined on $[0, T]$ with values in the space $V$.

Further, we can define the bilinear and bounded operator $B: V \times V \rightarrow V$ by the uniquely solved equation

$$
\langle H(0) B(y, w), \phi\rangle=\iint_{\Omega}[y, w] \phi d x \text { for all } y, w, \phi \in V
$$

The equation (23) can then be expressed as the Volterra integral equation of the second kind in the Hilbert space $V$ :

$$
\Phi(t)-\int_{0}^{t} K(t-s) \Phi(s) d s=-h B\left(\partial_{t} y(t), y(t)\right),
$$

where $K(t): V \rightarrow V$ are the operators defined by

$$
K(t)=-H(0)^{-1} \partial_{t} H(t), \quad t>0
$$


The equation (28) has due to the theory of Volterra integral equations (Balakrishnan,1976) a unique solution $\Phi \in L^{2}(0, T ; V)$, which can be expressed in a form

$\Phi(t)=-h B\left(\partial_{t} y(t), y(t)\right)-h \int_{0}^{t} M(t, s) B\left(\partial_{s} y(s), y(s)\right) d s$

$M(t, s): V \rightarrow V$ is the iterated kernel defined as the series of

$M(t, s)=\sum_{n=1}^{\infty} K_{n}(t, s)$

of the iterated kernels

$$
\begin{aligned}
& K_{n}(t, s)=\int_{s}^{t} K(t-\sigma) K_{n-1}(\sigma, s) d \sigma, \\
& K_{1}(t, s)=K(t-s)
\end{aligned}
$$

Inserting the Airy stress function $\Phi$ from (30) into (24) we arrive at the canonical initialboundary value problem for the determining the deflection function $y:[0, T] \rightarrow V$ :

$$
\begin{aligned}
& A_{1} \partial_{t} y(t)+A_{0} y(t)+h\left[B\left(\partial_{t} y(t), y(t)\right), y(t)\right]+h\left[\int_{0}^{t} M(t, s) B\left(\partial_{s} y(s), y(s)\right) d s, y(t)\right]=v(t)(34) \\
& y(0)=0
\end{aligned}
$$

Using the method of elliptic regularization (Lions,1969) the problem (34), (35) can be solved in the spaces of Bochner integrable functions

$\mathcal{V}=L^{2}(0, T ; V), \quad \mathcal{V}^{*}=L^{2}\left(0, T ; V^{2}\right)$,

$\mathcal{W}=\left\{y \in \mathcal{V}: \partial_{t} y \in \mathcal{V}, y(0)=0\right\}$

$\mathcal{V}$ is the Hilbert space with the inner product

$((u, v))=\int_{0}^{T}(u(t), v(t))_{V} d t$

We express the initial value problem (34), (35) in the operator form

$\mathcal{A}(y)=v, \quad y \in \mathcal{W}, \quad v \in L^{2}\left(0, T ; L^{2}(\Omega)\right) \subset \mathcal{V}^{*}$

where the operator $\mathcal{A}: \mathcal{W} \rightarrow \mathcal{V}^{*}$ is defined by

$$
\begin{aligned}
& \langle\langle\mathcal{A}(y), w\rangle\rangle=\int_{0}^{T}\left\langle A_{1} \partial_{t} y(t)+A_{0} y(t), w(t)\right\rangle d t+ \\
& +h \int_{0}^{T}\left(\left[B\left(\partial_{t} y(t), y(t)\right)+\int_{0}^{t} M(t, s) B\left(\partial_{s} y(s), y(s)\right) d s, y(t)\right], w(t)\right) d t, \quad y \in \mathcal{W}, \quad w \in \mathcal{V} .
\end{aligned}
$$


We introduce further the operators $L: \mathcal{W} \rightarrow \mathcal{V}$ and $\mathcal{A}_{\varepsilon}: \mathcal{W} \rightarrow \mathcal{W}^{*}$ by

$L y=\partial_{t} y, \quad y \in \mathcal{W}$

$\left\langle\left\langle\mathcal{A}_{\varepsilon}(y), w\right\rangle\right\rangle=\varepsilon((L y, L w))+\langle\langle A(y), w\rangle\rangle, \quad y, w \in \mathcal{W}$.

$\mathcal{A}_{\varepsilon}\left(y_{\varepsilon}\right)=v$

The operator $\mathcal{A}_{\varepsilon}: \mathcal{W} \rightarrow \mathcal{W}^{*}$ is coercive and pseudomonotone and then there exists for every $v \in \mathcal{W}^{*}$ a solution $y_{\varepsilon} \in \mathcal{W}$ of (43). The convolutive positivity (26) plays the crucial role in the coercivity argument.

After the limit procedure we obtain the existence theorem for the canonical form of the original problem. The theorem has been more detaily verified in (Bock, to appear).

Theorem 1 For every $v \in L^{2}\left(0, T ; L^{2}(\Omega)\right)$ there exists a solution $y \equiv y(v) \in \mathcal{W}$ of the problem (34), (35) or (39).

\section{OPTIMAL CONTROL PROBLEMS}

Let $U_{\text {ad }} \subset L^{2}\left(0, T ; L^{2}(\Omega)\right)$ be an arbitrary convex closed and bounded set of admissible controls. We introduce the cost functional $J: \mathcal{W} \times U_{a d} \rightarrow R$ of the form

$J(y, v)=\mathcal{J}(y)+j(v), \quad y \in \mathcal{W}, \quad v \in U_{a d}$

We shall investigate the following

Optimal Control Problem $\mathcal{P}$ : To find a couple $\left(y_{0}, u\right) \in \mathcal{W} \times U_{a d}$ such that

$J\left(y_{0}, u\right)=\min _{(y, v) \in \mathcal{K}} J(y, v)$,

where

$\mathcal{K}=\left\{(y, v) \in \mathcal{W} \times U_{a d}: \mathcal{A}(y)=v\right\}$.

Theorem 2 If the functionals $\mathcal{J}, j$ are weakly lower semicontinuous on $W$ and $L^{2}(0, T ; L(\Omega))$ respectively, then there exists a solution $\left(y_{0}, u\right) \in \mathcal{K}$ of the Optimal Control Problem $\mathcal{P}$.

Proof. A solution is a weak limit of a minimizing sequence $\left\{y_{n}, u_{n}\right\}$ for a functional $J$ on $\mathcal{K}$. This limit exists due to the boundedness in $\mathcal{V}$ of sequences $\left\{y_{n}\right\}$ and $\left\{\partial_{t} y_{n}\right\}$ of solutions and their derivatives corresponding to a bounded sequence $\left\{u_{n}\right\} \subset U_{a d}$.

The operator $\mathcal{A}: \mathcal{W} \rightarrow \mathcal{V}^{*}$ is Fréchet differentiable with the derivative $\mathcal{A}^{\prime}(y) \in$ $\mathcal{L}\left(\mathcal{W}, \mathcal{V}^{*}\right)$ defined by

$$
\left\langle\mathcal{A}^{\prime}(y) z, w\right\rangle_{\mathcal{V}}=\int_{0}^{T}\left\{\left\langle A_{1} \partial_{t} z+A_{0} z, w\right\rangle+\right.
$$


$+h\left(\left[B\left(\partial_{t} y, y\right)+\int_{0}^{t} M(t, s) B\left(\partial_{s} y(s), y(s)\right) d s, z(t)\right]+\right.$

$\left.\left.+\left[\partial_{t} B(y, z)+\int_{0}^{t} M(t, s) \partial_{s} B(y(s), z(s)) d s, y(t)\right], w(t)\right)_{0}\right\} d t$,

where $(y, w)=\iint_{\Omega} y \cdot w d \Omega$.

Let us define the set $\mathcal{W}_{T}=\left\{w \in \mathcal{V}: \partial_{t} w \in V, w(T)=0\right\}$. The adjoint operator $\mathcal{A}^{\prime}(y)^{*}: \mathcal{W}_{T} \rightarrow \mathcal{V}^{*}$ has then the form

$\left\langle\mathcal{A}^{\prime}(y)^{*} w, z\right\rangle_{\mathcal{V}}=\int_{0}^{T}\left\{\left\langle-A_{1} \partial_{t} w+A_{0} w, z\right\rangle+\right.$

$+h\left(\left[B\left(\partial_{t} y, y\right)+\int_{0}^{t} M(t, s) B\left(\partial_{s} y(s), y(s)\right) d s, w(t)\right]-\right.$

$\left.\left.-\left[\partial_{t} B(y, w)+\partial_{t} \int_{t}^{T} M^{*}(s, t) B(y(s), w(s)) d s, y(t)\right], z(t)\right)_{0}\right\} d t$.

Using the general extremal principle in smoothly convex problems (Joffe and Tichomirov, 1984) we obtain the following necessary optimality conditions:

Theorem 3 Let the couple $\left(y_{0}, u\right) \in \mathcal{W} \times U_{a d}$ be a solution of the Optimal Control Problem $P$ with a convex functional $j$ and continuously Fréchet differentiable functionals $\mathcal{J}, j$. Then there exists an element $p \in \mathcal{W}_{T}$ such that

$\mathcal{A}\left(y_{0}\right)=u$

$\mathcal{A}^{\prime}\left(y_{0}\right) p=-\mathcal{J}^{\prime}\left(y_{0}\right)$

$\int_{0}^{T}\left(j^{\prime}(u)-p, v-u\right) d t \geq 0 \quad$ for all $\quad v \in U_{a d}$

Using the method of penalization an Optimal Control Problem with a cost functional involving all solutions of the state problem can be solved.

Let $\mathcal{F}: \mathcal{W} \times U_{a d} \rightarrow \mathcal{V}^{*}$ be the state operator of the form $\mathcal{F}(y, v)=\mathcal{A}(y)-v$. We introduce the cost functional

$\hat{J}(v)=\sup _{y \in \mathcal{W}, \mathcal{F}(y, v)=0}[\mathcal{J}(y)+j(v)], \quad v \in U_{a d}$.

In an analogous way as in the case of stationary Von Kárman's equations (Bock, Hlaváček and Lovíšek, 1987) there can be verified the existence of an optimal control $u \in U_{a d}$ fulfilling $\hat{J}(u)=\min _{v \in U_{a d}} \hat{J}(v)$. The element $u$ is a weak limit of a subsequence $\left\{u_{\varepsilon_{n}}\right\} \subset U_{a d}$ fulfilling $\lim _{n \rightarrow \infty} \varepsilon_{n}=0, \varepsilon_{n}>0$ and

$J_{\varepsilon}\left(u_{\varepsilon}\right)=\min _{v \in U_{a d}} J_{\varepsilon}(v)$, 
$J_{\varepsilon}(v)=\sup _{y \in \mathcal{W}_{r}}\left[\mathcal{J}(y)+j(v)-\frac{1}{\varepsilon}\|\mathcal{F}(y, v)\|_{\nu^{*}}\right]$

$\mathcal{W}_{r}=\left\{y \in \mathcal{W}:\|y\|_{\mathcal{W}} \leq r\right\}$,

where $r>0$ is chosen such that for every $v \in U_{a d}$ we have $y(v) \in \mathcal{W}_{r}$.

\section{REFERENCES}

Balakrishnan, A.V.(1976) Applied functional analysis. Springer Verlag, New York.

Bock, I.(to appear) Von Kárman's equations for viscoelastic plates. ZAMM.

Bock, I., Hlaváček,I. and Lovíšek,J. (1984) On the optimal control problems governed by the equations of von Kárman, I. The homogeneous Dirichlet boundary conditions. Applications of math., 29, 303-314.

Bock, I., Hlaváček, I. and Lovíšek, J. (1985) On the optimal control problems governed by the equations of von Kárman, II. Mixed boundary conditions. Applications of math., 30, 375-392.

Bock, I., Hlaváček, I. and Lovíšek, J. (1987) On the optimal control problems governed by the equations of von Kárman, III. The case of an arbitrary large perpendicular load. Applications of math., 32, 315-331.

Brilla, J. (1973) Variational methods in mathematical theory of viscoelasticity. In: Ráb,M.; Vosmanský,J.(eds.): Proc. Internat. Conf. on Diff. Equations, Equadiff III. University J.E.Purkynè Press, Brno, 211-216.

Christensen, R.V. (1971) Theory of viscoelasticity. Academic Press, New York.

Joffe, A.D. and Tichomirov, V.M. (1984) The theory of extremal problems (in Russian). Nauka, Moskva.

Lions, J.L. (1969) Quelques méthodes de résolution des problèmes aux limites non linéaires. Dunod, Paris .

MacCamy, R.C. and Wong, J.S.W. (1972) Stability theorems for some functional equations. Transactions of AMS 164,1, 1-37. 